\title{
Cryo-Electron Microscopy of Potassium Channel Membrane Proteins
}

\author{
Julia Kowal $^{1}$, Sebastian Scherer ${ }^{1}$, Kushal Sejwal ${ }^{1}$, Mohamed Chami ${ }^{1}$, Paul Baumgartner ${ }^{1}$, Martina \\ Rangl $^{2}$, Simon Scheuring ${ }^{2}$, Gunnar F. Schröder ${ }^{3}$, Crina Nimigean ${ }^{4}$, and Henning Stahlberg ${ }^{1}$ \\ a \\ 1. Center for Cellular Imaging and NanoAnalytics (C-CINA), Biozentrum, University of Basel, \\ Mattenstrasse 26, CH-4058 Basel, Switzerland \\ 2. U1006 INSERM, Aix-Marseille Université, Parc Scientifique et Technologique de Luminy, 163 \\ Avenue de Luminy, 13009 Marseille, France \\ 3. Forschungszentrum Jülich, Institute of Complex Systems, ICS-6: Structural Biochemistry, 52425 \\ Jülich; and Department of Physics, Heinrich-Heine University Düsseldorf, 40225 Düsseldorf, Germany \\ 4. Departments of Anesthesiology, Physiology and Biophysics, and Biochemistry, Weill Cornell Medical \\ College, 1300 York Ave, New York, New York 10065, USA
}

Membrane proteins are central to health and disease. A detailed understanding of the function of membrane proteins requires access to high-resolution structural data and mechanistic insight into conformational changes occurring when the proteins are active. The classical structure determination method, X-ray crystallography, is challenged by membrane proteins, which are both difficult to purify in large amounts and difficult to crystallize in three dimensions. Electron crystallography requires less material than X-ray methods and allows the study of membrane proteins in their membrane embedded state. The purified, detergent solubilized proteins are reconstituted into artificial phospholipid membranes; highly ordered two dimensional (2D) membrane protein crystals are obtained by carefully tuning the buffer conditions during controlled detergent dialysis.

We obtained well-ordered, large 2D crystals of full-length MloK1, a cyclic nucleotide-modulated potassium channel from Mesorhizobium loti, in the presence and absence of its ligand, cAMP. Using cryo-electron microscopy we determined three-dimensional (3D) maps at $7 \AA$ resolution in the membrane plane and $12 \AA$ resolution in the vertical direction [1]. A mechanism of channel gating upon cyclic nucleotide binding was formulated from the observed differences between the two models in the presence and absence of cAMP. The cryo-EM structures presented here provide new insight into the arrangement of the cyclic nucleotide-binding domains in the context of the full-length channel in the presence and absence of ligand.

In cases where flat cryo-preparations of well-ordered 2D crystals are available, electron crystallography is a powerful method for the determination of the high-resolution structures of membrane and soluble proteins. However, crystal unbending and Fourier filtering methods in electron crystallography 3D image processing are generally limited in their performance for $2 \mathrm{D}$ crystals that are badly ordered or non-flat. A single particle image processing approach for the determination of high-resolution 3D structures of membrane proteins [2] addresses the low signal-to-noise ratio (SNR) of 2D crystal images by exploiting neighbourhood correlation between adjacent proteins in the $2 \mathrm{D}$ crystal. We successfully applied the new refinement method to the structure of the potassium channel MloK1.

In parallel, we developed a new automation pipeline ( $2 \mathrm{dx}$ automator, [3]), which provides real-time image processing results to the operator of the electron microscope. The pipeline allows the microscope operator to focus on the recording of highest resolution data, while fully eliminating the need for manual image processing. The new processing pipeline exploits the high SNR and frame readout frequency of 
direct electron detectors to automatically correct for beam-induced sample movement, and reliably processes individual crystal images without human interaction. With this new pipeline an automatically generated unit cell projection map of each recorded 2D crystal is available in less than 5 minutes after the image was recorded. The results obtained are superior to those delivered by conventional photographic film-based methodology of the same sample described above, and demonstrate the importance of drift-correction (see Figure 1).

Additionally, we propose to employ the recently established cryo-electron microscopy imaging capabilities and our newly developed image processing software, to study the dynamic structure of selected membrane protein systems in liposomes, a close-to-native environment.

In conclusion, we are employing a combination of cryo-electron crystallography, single particle processing of $2 \mathrm{D}$ crystals [2] and $2 \mathrm{dx}$ _automator processing pipeline [3] to determine the 3D structures of selected systems. The produced data complement existing high-resolution X-ray structural studies with information about the dynamic functioning of these classes of membrane proteins.

\section{References:}

[1] Kowal et al, Nat Commun. 2014 Jan 28;5:3106.

[2] Scherer et al, J Struct Biol. 2013 Dec 29. pii: S1047-8477(13)00338-9.

[3] Scherer et al, 2dx_automator: Implementation of a Semiautomatic High-Throughput

High-Resolution Cryo-Electron Crystallography Pipeline, 2014, Submitted.

[4] This work was supported by the Swiss National Science Foundation (SNF 315230_127545, National Centers for Competence in Research, Structural Biology and TransCure), the Swiss Initiative for Systems Biology (SystemsX.ch), the National Institutes of Health (NIH GM077560 to C.M.N.), the European Research Council (Grant \#310080 to S.S.), and Agence National de la Recherche (ANR, Grants \# ANR-12-BS10-009-01 and ANR-12-BSV8-0006-01 to S.S.). We acknowledge expert technical assistance of B. Anderson and K. Goldie. We thank S.A. Müller for insightful discussions and manuscript editing.
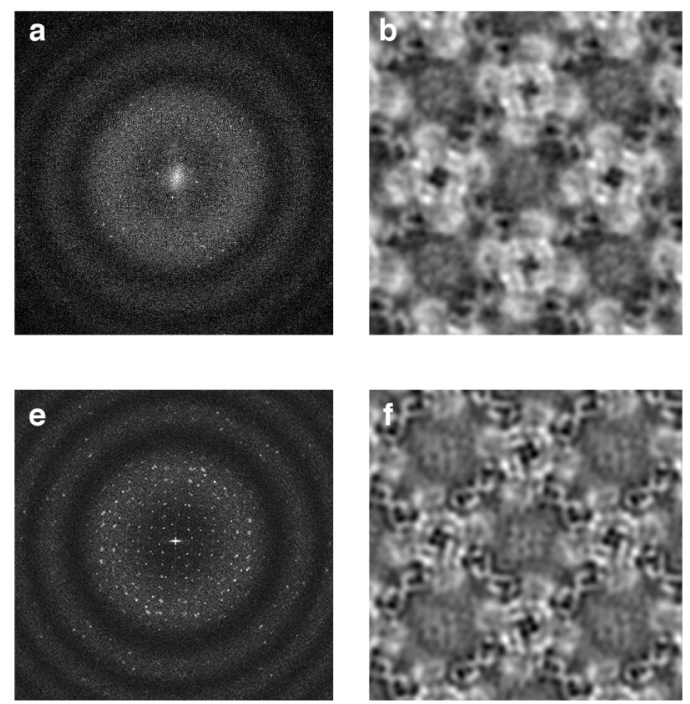
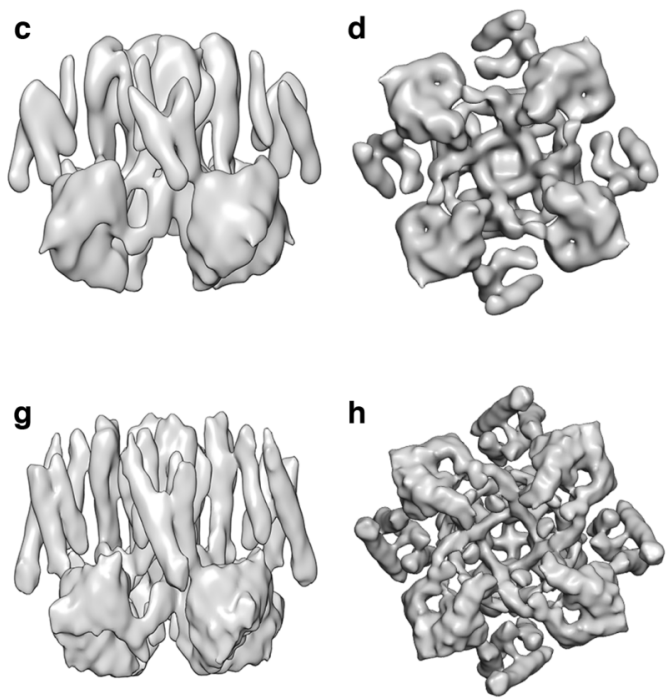

Figure 1. Impact of direct electron detectors and drift-correction. (a) Power spectrum of an image recorded on film. (b) Merged unit-cell obtained from one 2D crystal of MloK1. (c) Side view and (d) top view of the film data 3D reconstruction. (e-h) Direct electron detector data with drift-correction. 